\title{
No job too small
}

\section{The collective approach to science at the nanoscale.}

To enable, control and monitor processes at the nanoscale the development of matching imaging and sensing technologies is essential. This month we highlight recent advances in highresolution imaging facilitated by surface plasmons. This collective motion of electrons at the surface of metals enables imaging capabilities far beyond the diffraction limit of classical free-space optics, with intriguing new applications.

The main limitation to the operation of classical optical instruments is diffraction - the bending of waves around an obstacle due to the interference of different light paths reflected from it. Diffraction is a direct consequence of the wave properties of light. The first comprehensive report on diffraction was by Francesco Maria Grimaldi, who observed that sunlight passing through a small hole in a screen forms a cone with a propagation angle larger than expected if a rectilinear propagation of light is assumed ${ }^{1}$. Similarly, the edges of the light cone are fuzzy and show coloured fringes. Importantly, the influence of diffraction places a lower limit on the smallest structure an optical instrument can resolve to roughly the wavelength of light used in imaging.

However, as diffraction arises from the wave properties of light, these imaging limitations can be overcome by fundamentally different approaches, some of which are outlined in the Commentary by Nikolay Zheludev². For example, thin-film nanohole arrays may lead to lithography with super-resolution.

A particularly successful imaging approach is based on the use of surface plasmons. Key to these imaging applications is the strong coupling of surface plasmons to light waves of a matching frequency. The combined photon-plasmon entity is also referred to as a surface plasmon polariton.

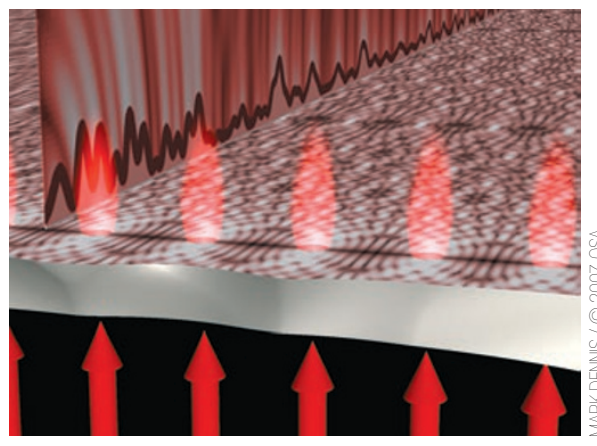

the Review Article by Jeffery Anker and colleagues, their functionality arises from molecules that are attached or close to gold nanoparticles or surfaces ${ }^{4}$. If the surface-plasmon resonance matches that of the molecule, the metal nanoparticles act as antennas whose strong signal enhancement makes them extremely useful and versatile sensors for use in immunoassays, in living cells or for spectroscopic applications.

The advances in the area of imaging and sensing enabled by plasmonic effects demonstrate how collective

Surface plasmon effects. A light beam (red) that hits an array of nanoholes excites surface plasmons. The intensity variations of the plasmons across the plane show, in analogy to the classical Talbot effect, that the plasmon pattern around the holes is repeated at regular intervals ${ }^{5}$. This effect may, in conjunction with plasmon waveguides, be used for focusing.

Effectively, at the surface-plasmon resonance frequency, local light fields may be strongly enhanced. Indeed, one of the applications of surface plasmons is their use as plasmonic waveguides, where, for example, light is guided across a chain of silver nanoparticles.

In imaging, the surface-plasmon resonance of thin silver films can be successfully used to capture and enhance light emitted from a nearby object. As Xiang Zhang and Zhaowei Liu describe in their Progress Article, this allows the realization of so-called superlenses and hyperlenses with imaging resolution beyond the diffraction limit ${ }^{3}$. Although superlenses bring microscopy down to the nanoscale as long as the imaging objects are close to the lens, their potential for applications may also lie in their capability for high-resolution lithography.

Surface plasmons are also promising for biosensors. Explained in detail in phenomena at the nanoscale can be used for emerging applications that challenge the existing limits of the macro and mesoscale. Indeed, surface plasmons may represent just one particularly successful demonstration of this trend, and other effects may appear as condensed-matter physics ventures into the nanoscale. For example, recent experiments show that thin films and nanoparticles of magnetite can undergo a phase transition otherwise unknown for bulk samples of magnetite.

As for applications, the example of superlenses and plasmonic nanoparticles obviously suggest that, as the length scales we investigate become smaller, we have to look for physics intrinsic to these length scales to truly realize the potentials offered by nanotechnology. The advances in imaging capabilities highlighted in this issue certainly provide a compelling visual demonstration of the many opportunities offered by collective phenomena at the nanoscale.

\section{References}

1. Grimaldi, F. M. Physicomathesis de lumine, coloribus, et iride, aliisque annexis. (Bologna, 1665).

2. Zheludev, N. I. Nature Mater. 7, 420-422 (2008).

3. Zhang, X. \& Liu, Z. Nature Mater. 7, 435-441 (2008).

4. Anker, J. N., Hall, W. P., Lyandres, O., Shah, N. C., Zhao, J. \& Van Duyne, R. P. Nature Mater. 7, 442-453 (2008).

5. Dennis, M. R., Zheludev, N. I. \& García de Abajo, F. J. Opt. Expr. 15, 9692-9700 (2007). 